\title{
Médiévales
}

Langues, Textes, Histoire

77 | automne 2019

Mathématiques. Savoirs et enseignements (OrientOccident)

\section{Mathématiques au Moyen Âge : savoirs, langues et enseignements}

Marc Moyon

\section{OpenEdition}

\section{Journals}

Édition électronique

URL : https://journals.openedition.org/medievales/10192

DOI : $10.4000 /$ medievales. 10192

ISSN : 1777-5892

Éditeur

Presses universitaires de Vincennes

Édition imprimée

Date de publication : 5 décembre 2019

Pagination : 5-9

ISBN : 978-2-37924-061-4

ISSN : 0751-2708

Référence électronique

Marc Moyon, "Mathématiques au Moyen Âge : savoirs, langues et enseignements », Médiévales [En ligne], 77 | automne 2019, mis en ligne le 01 janvier 2022, consulté le 22 avril 2022. URL : http:// journals.openedition.org/medievales/10192 ; DOI : https://doi.org/10.4000/medievales.10192 
Marc Moyon

\section{Mathématiques au Moyen Âge : savoirs, langues et enseignements}

Ce volume de Médiévales est le fruit de diverses rencontres entre historiens des mathématiques médiévales et autres historiens médiévistes ${ }^{1}$. S'il n'est plus essentiel de démontrer toute l'utilité du Moyen Âge pour l'étude historique des sciences - et des mathématiques en particulier -, il n'en est pas moins précieux de donner à voir la richesse des études actuelles menées sur les mathématiques du Moyen Âge, le renouvellement des problématiques en prenant davantage en compte les contextes de production et de circulation des savoirs, les nouvelles approches méthodologiques et interrogations sur les textes eux-mêmes, tant dans leur contenu scientifique que dans leur organisation ou leur forme matérielle, qu'ils soient connus ou inédits. C'est toute l'ambition des six contributions rassemblées dans ce volume thématique francophone ${ }^{2}$.

S'intéresser aux mathématiques médiévales n'est pas élémentaire, au-delà même de la seule technicité apparente de la discipline. Mais, si comprendre les mathématiques des textes disponibles est indispensable, c'est bien loin de suffire. Les difficultés sont aussi ailleurs. Tout médiéviste sait que la documentation est lacunaire et exprimée dans des langues souvent difficiles à lire et à comprendre. Les contextes de production et de

1. Le projet éditorial proposé par le comité de rédaction de Médiévales a largement bénéficié, entre autres, des travaux menés dans le cadre de la journée d'étude " Des mathématiques médiévales : savoirs, textes, langues et enseignement» (16 mars 2018) organisée dans le cadre du séminaire d'histoire des mathématiques de l'Institut Henri Poincaré, avec le soutien du GdR 3398 « Histoire de mathématiques » du CNRS. Je tiens à remercier ici les membres du comité de rédaction de Médiévales, et en particulier Christopher Lucken pour son implication dans le suivi de ce dossier thématique, ainsi que l'ensemble des participant·e·s à la journée pour leurs questions et leurs remarques.

2. Nous pourrions considérer que le présent volume complète le numéro 52 (2007) de Médiévales, sur " La science médiévale, du codex à l'imprimé », dirigé par L. Moulinier et $\mathrm{N}$. Weill-Parot, dans lequel les mathématiques étaient formellement absentes. 
circulation du savoir ne sont pas toujours historiquement bien documentés. Le travail sur les manuscrits, qui restent la principale source du médiéviste, requiert des connaissances et des compétences diverses et complémentaires, entre autres dans les domaines de la paléographie, de la codicologie ou encore de la philologie.

L'ensemble des études réunies ici contribue donc à mettre en lumière un Moyen Âge mathématique, entre les pays d'Islam et l'Europe latine, principalement entre les $\mathrm{XII}^{\mathrm{e}}$ et $\mathrm{XIV}^{\mathrm{e}}$ siècles, où les textes et leur histoire, les langues d'expression et les relations qu'elles entretiennent entre elles sont interrogés au même titre que les savoirs. Enfin, prolongeant les premières réflexions de Guy Beaujouan à propos du quadrivium (et particulièrement de l'arithmétique) ${ }^{3}$, ces études donnent aussi à voir l'importance de l'enseignement - et de ses différents contextes, institutionnels ou non - dans la production et la circulation des mathématiques. Aujourd'hui, l'historien des mathématiques médiévales ne peut pas l'ignorer.

Sonja Brentjes propose un éclairage sur plusieurs points historiographiques liés à l'histoire des sciences exactes, et plus particulièrement des mathématiques, en pays d'Islam. Elle interroge la manière avec laquelle est étudié l'ensemble des textes mathématiques élémentaires produits en pays d'Islam, sur une longue période ${ }^{4}$ en centrant son argumentaire sur le Moyen Âge. Les historiens des mathématiques des pays d'Islam ont d'abord ignoré ces textes, privilégiant les traités offrant de nouveaux résultats ou de nouvelles méthodes. Aussi, les recherches en histoire des mathématiques se sont principalement concentrées sur le contenu, abandonnant ainsi les autres aspects transmis par ces textes comme leurs formes matérielles, leurs organisations, les usages... S. Brentjes estime nécessaire, lorsqu'on étudie l'enseignement des mathématiques en pays d'Islam, d'intégrer pleinement l'analyse de ces textes élémentaires et de les inscrire plus largement dans leur contexte de production et de transmission. Elle les considère comme des témoins d'un système éducatif qui a commencé à inclure les sciences mathématiques à partir du $\mathrm{XII}^{\mathrm{e}}$ siècle dans et en dehors des grands centres urbains. C'est ainsi qu'est mis l'accent sur des textes complémentaires aux premiers - les certificats d'enseignement, les dictionnaires biographiques et plus généralement

3. Citons, entre autres, G. Beaujouan, «L'enseignement du "Quadrivium" », dans La scuola nell'Occidente latino dell'alto medioevo, Spolète, 1972 (Settimane di studio del Centro italiano di studi sul'alto medioevo, XIX), t. II, p. 639-723 ; ID., « Le Quadrivium et la faculté des Arts », dans O. WeiJers et L. Holtz éd., L'Enseignement des disciplines à la faculté des arts (Paris et Oxford, XIII'-XIV siècles), Turnhout, 1997, p. 185-194.

4. Elle mène une étude plus large dans S. BRENTJES, Teaching and Learning the Sciences in Islamicate Societies (800-1700) (Studies on the Faculty of Arts. History and Influence), Turnhout, 2018. 
les éléments paratextuels -, envisagés comme de nouvelles sources pour décrire le paysage de l'enseignement des sciences exactes en pays d'Islam.

L'article d'Eleonora Sammarchi, plus technique, nous plonge dans l'œuvre d'al-Zanjān̄̄, mathématicien persan de la première moitié du XIII ${ }^{\mathrm{e}}$ siècle, avec son Qisțās al-mu'ādala fì 'ilm al-jabr wa'l-muqābala [Balance de l'équation sur la science de la restaurationet de la comparaison]. Inscrit notamment dans la filiation des travaux d'al-Karajī ( $\mathrm{x}-\mathrm{XI}^{\mathrm{e}}$ siècles), qui visent à explorer les rapports entre arithmétique et algèbre ${ }^{5}$, cet ouvrage est composé d'une partie théorique explicitant l'algèbre avec ses objets et ses opérations caractéristiques, et de deux collections de problèmes qui en comptent au total plus de trois cents. Ce sont ces collections qui sont au cœur du travail d'E. Sammarchi ${ }^{6}$. La mise en série des problèmes fait d'abord émerger des marqueurs de transmission à travers la tradition algébrique arabe, soit explicitement, soit implicitement ${ }^{7}$. L'étude d'un échantillon de problèmes donne ensuite à voir le calcul algébrique en action avec ses nouvelles préoccupations. Ainsi, les problèmes d'analyse indéterminée ${ }^{8}$ tendent à se constituer progressivement en nouveau chapitre au sein de l'algèbre. L'auteure défend enfin l'idée que les problèmes permettent de comprendre pourquoi et comment appliquer les règles du calcul algébrique.

L'algèbre est vue au Moyen Âge comme un art nouveau pour résoudre des problèmes. Cette discipline, née entre 813 et 833 à Bagdad avec al-Khwārizmī, est progressivement assimilée par les Latins à partir du $\mathrm{XII}^{\mathrm{e}}$ siècle, en particulier grâce aux traductions latines réalisées en Andalus à partir de l'arabe. Marc Moyon revient sur cette appropriation latine grâce à l'étude d'un corpus arabo-latin, probablement dû au célèbre traducteur Gérard de Crémone (mort vers 1187), regroupant quatre textes de mathématiques élémentaires, présentés comme des compilations de problèmes. Leur objectif commun est la résolution de problèmes linéaires et quadratiques ${ }^{9}$, qu'ils soient de nature géométrique ou arithmétique. L'organisation de

5. Plus largement, sur cette question, voir R. RASHED, Entre arithmétique et algèbre, Paris, 1984.

6. Cet article, ainsi que les deux suivants, illustrent les questions débattues au sein du projet «Séries de problèmes : un genre au croisement des cultures » du laboratoire d'excellence HASTEC [en ligne] https://problemata.hypotheses.org [consulté le 17 juillet 2019]. Voir l'ouvrage collectif dont une partie traite du Moyen Âge, « Les séries de problèmes, un genre au carrefour des cultures : une première synthèse », SHS Web of Conferences, 22 (2015).

7. Dans son étude comparative, E. Sammarchi considère trois auteurs parmi les plus importants : le père fondateur de l'algèbre al-Khwārizmī (mort vers 850), Abū Kāmil (mort vers 930) et enfin al-Karajī.

8. L'analyse indéterminée est la branche des mathématiques consacrée à la résolution des équations diophantiennes (équations polynomiales à coefficients et à solutions entières).

9. Les problèmes linéaires sont des problèmes du premier degré. Ce sont des problèmes qui, algébriquement, se ramènent à la résolution d'équations du type $a x=b$. Les problèmes quadratiques (ou plans), quant à eux, sont des problèmes du second degré : ils se ramènent à la résolution d'équations faisant intervenir le carré de l'inconnu. 
ces compilations montre en réalité une double mise en série générée à la fois par la thématique des problèmes et par une variation des paramètres mathématiques. L'algèbre apparaît là comme une méthode alternative à bien d'autres, plus archaïques, comme des procédures algorithmiques ou encore des méthodes de fausse position. Si l'étude philologique et celle du contenu sont naturellement indispensables, la codicologie est aussi utilisée pour montrer la stabilité du corpus et pour formuler quelques hypothèses sur l'histoire des textes, de leur production à leur utilisation. En particulier, M. Moyon formule des hypothèses montrant l'éventuelle utilisation de ce corpus à des fins d'enseignement - dont les modalités restent à définir - des mathématiques élémentaires héritées des pays d'Islam.

Stéphane Lamassé nous présente une vaste étude de problèmes mathématiques considérés comme « récréatifs »: ils peuvent être, entre autres, des cautelae, des subtilitates ou encore des enigmata. Dans la tradition textuelle, ces problèmes sont regroupés en collection ou apparaissent isolément. Ils traversent le Moyen Âge et jouissent, dès le IX siècle, d'une importante diffusion, notamment dans le cadre d'une transformation de l'enseignement des écoles cathédrales vers l'université. Dans cette contribution, S. Lamassé dresse une synthèse historiographique sur ce corpus caractéristique des mathématiques médiévales, où il montre - et regrette - que les historiens ont obstinément étudié chaque problème comme une entité textuelle indépendante de ceux qui l'entoureraient, en négligeant la plupart du temps son environnement codicologique. À partir de cette synthèse et en se fondant sur une méthodologie adaptée, un ensemble de recueils de problèmes peut être isolé. Il s'agit alors de décrire pas à pas leur cohérence que seule l'étude des séries permet de repérer. Cette cohérence explicitée dans une perspective diachronique amène à conjecturer quels sont les éventuels usages des recueils étudiés ici.

À travers une étude de type philologique, Nicoletta Rozza offre un certain nombre de remarques lexicales centrées sur la Practica geometriae [Pratique de la géométrie] de Léonard de Pise (mort après 1241), autrement connu sous le nom de Fibonacci (Filius Bonacii). L'auteure s'intéresse de près au texte pour tenter de percer des éléments de la langue en cours de spécialisation du mathématicien latin. Les premiers résultats montrent l'importance du lexique de l'arpentage du haut Moyen Âge et du corpus arabo-latin de la géométrie de la mesure ${ }^{10}$. Si Fibonacci semble bien connu de tout historien des mathématiques, très peu d'études spécialisées sont menées sur les textes fibonacciens eux-mêmes, qu'il s'agisse des œuvres majeures, comme le Liber abaci [Livre du calcul] et la Practica geometriae, ou des œuvres mineures, comme le Flos [Fleur] et le Liber quadratorum

10. Voir plus largement M. Moyon, La Géométrie de la mesure dans les traductions arabo-latines médiévales, Turnhout, 2017. 
[Livre des < nombres > carrés]. Cela rend l'étude ponctuelle de N. Rozza encore plus précieuse pour tout médiéviste s'intéressant à des textes techniques latins.

Avec la dernière contribution de ce volume consacré aux mathématiques, nous quittons la République maritime de Pise pour l'université parisienne. Sabine Rommevaux-Tani nous amène précisément à la faculté des Arts de Paris, aux XIII-XIV ${ }^{\mathrm{e}}$ siècles, avec Radulphus Brito ou Raoul le Breton (mort en 1320/21). Maître ès arts, auteur d'un commentaire des Sentences de Pierre Lombard (mort en 1160), Raoul le Breton est, entre autres, l'auteur d'un traité de philosophie des mathématiques qui pourrait être le reflet de son enseignement à la faculté des Arts. Comme le requiert la tradition universitaire médiévale ${ }^{11}$, cet enseignement est organisé en questiones, consacrées ici à des propos généraux sur le quadrivium, à l'arithmétique, à la géométrie, au comput et à la musique. S. RommevauxTani étudie d'abord la tradition manuscrite de ces Questiones mathematice, en donne ensuite une description générale, pour révéler enfin des éléments de la réflexion ontologique de Raoul autour de l'unité et du nombre.

Nous espérons que ce numéro thématique de Médiévales donnera envie aux lecteur·rice's de voir se poursuivre le dialogue entre médiévistes et historien'ne's des mathématiques. Saluons pour finir l'effort des six auteur·e's, qui ont suivi la requête de Danielle Jacquart ${ }^{12}$ en faisant " preuve de pédagogie à l'égard de [leurs] collègues non-spécialistes et [en] ne [cédant pas] à la tentation d'isoler l'exposé scientifique du contexte dans lequel il s'insère, ni de le réduire aux démonstrations les plus convaincantes ».

Marc Moyon - Université de Limoges - CNRS, XLIM, UMR 7252

11. Voir, par exemple O. WeIJERs, «L'enseignement du trivium à la Faculté des arts de Paris : la "questio" », dans J. HAMESSE éd., Manuels, programmes de cours et techniques d'enseignement dans les universités médiévales, Louvain-la-Neuve, 1994, p. 57-74.

12. D. JACQUART, «Quelle histoire des sciences pour la période médiévale antérieure au XIII ${ }^{\mathrm{e}}$ siècle ? », Cahiers de civilisation médiévale, 153-154 (1996), «La recherche sur le Moyen Âge à l'aube du vingt-et-unième siècle », p. 97-113 (p. 112-113). 
